\title{
Ferromagnetic ordering in a generalized Hubbard model
}

\author{
L.Didukh, O.Kramar, Yu.Skorenkyy \\ Ternopil State Technical University, Department of Physics, \\ 56 Rus'ka Str., 46001 Ternopil, Ukraine
}

Received August 14, 2000

The present paper considers ferromagnetic ordering in the Hubbard model generalized by taking into account the inter-atomic exchange interaction and the correlated hopping in a partially filled narrow band. Expressions for the magnetization and for the Curie temperature as functions of model parameters and band filling are obtained in the case of weak intraatomic Coulomb interaction. The condition of ferromagnetic state realization is found. The results obtained indicate the important role of correlated hopping.

Key words: ferromagnetic ordering, correlated hopping, exchange interaction, magnetization, Curie temperature

PACS: $71.10 . F d, 71.30 .+h, 71.27 .+a$

\section{Introduction}

In spite of great attention paid to the investigation of ferromagnetism in narrow energy bands [1-7], where the same system of electrons is responsible both for conductivity and for magnetic ordering (the transition $3 d$-metals, ferromagnetic sulphides, oxides and selenides with a metallic type of conductivity) the problem still remains open $[8,9]$.

There are some experimental results for the disulphides of transition metals which can be interpreted on the basis of an itinerant mechanism of ferromagnetism. In these compounds the dependence of the Curie temperature on the number of magnetic moments is non-typical from the point of view of the exchange mechanism of ferromagnetism. In $\mathrm{Fe}_{x} \mathrm{Co}_{1-x} \mathrm{~S}_{2}$ [10] where the electron concentration $n$ changes from 0 to 1 in the doubly degenerate $e_{g}$-subband the Curie temperature increases with the decrease of the electron concentration at $0.75<n<1$.

Although the consistent theory of ferromagnetic ordering in transition metal compounds can be constructed only in a model including the orbital degeneracy of

*E-mail: didukh@tu.edu.te.ua 
the band, the qualitative character of the observed properties can be interpreted in the framework of a generalized model of a non-degenerate band [11].

\section{The Hamiltonian}

The simplest model for describing magnetic properties of narrow-band materials is the Hubbard model [12], but this model contains only diagonal matrix elements of the Coulomb interaction in the site representation, which do not give rise to metallic ferromagnetism except in special situations such as a single hole in a halffilled band [13] or a special lattice geometry [7]. The importance of the non-diagonal matrix elements of the Coulomb interaction was pointed out in references $[9,14]$.

We write down the Hamiltonian of a system of electrons in the following form [15, $16]$

$$
\begin{aligned}
H= & -\mu \sum_{i \sigma} a_{i \sigma}^{+} a_{i \sigma}+\sum_{i j \sigma}^{\prime} t_{i j}(n) a_{i \sigma}^{+} a_{j \sigma} \\
& +\sum_{i j \sigma}^{\prime}\left(T_{2}(i j) a_{i \sigma}^{+} a_{j \sigma} n_{i \bar{\sigma}}+\text { h.c. }\right)+U \sum_{i} n_{i \uparrow} n_{i \downarrow} \\
& +\frac{1}{2} J \sum_{i j \sigma \sigma^{\prime}}{ }^{\prime} a_{i \sigma}^{+} a_{j \sigma^{\prime}}^{+} a_{i \sigma^{\prime}} a_{j \sigma},
\end{aligned}
$$

where $a_{i \sigma}^{+}, a_{i \sigma}$ are creation and destruction operators of the electron on the site $i$, $\sigma=\uparrow, \downarrow, n_{i \sigma}=a_{i \sigma}^{+} a_{i \sigma}, n=\left\langle n_{i \uparrow}+n_{i \downarrow}\right\rangle, \mu$ is the chemical potential, $t_{i j}(n)=t_{i j}+n T_{1}(i j)$ is the effective hopping integral between the nearest neighbours, $t_{i j}$ is the hopping integral of an electron from site $j$ to site $i, T_{1}(i j)$ and $T_{2}(i j)$ are the parameters of correlated hopping [16] of the electron, $U$ is the intra-atomic Coulomb repulsion and $J$ is the exchange integral for the nearest neighbours. The primes on the sums in equation (2.1) signify that $i \neq j$.

The peculiarities of the model described by the Hamiltonian (2.1) take into consideration the influence of the site occupation on the electron hoppings (correlated hopping), and the exchange integral. The correlated hopping, firstly, renormalizes the initial hopping integral (it becomes concentration- and spin-dependent) and, secondly, leads to the shift of the band center independent on the quasiimpulse but dependent on magnetic ordering. Taking into account the quantity of the second order $J$ is in principle necessary to describe ferromagnetism in this model $[9,14,17]$. In this paper we do not take into account the inter-atomic Coulomb interaction, which is important in the charge ordering state (this ordering type goes beyond this article).

To characterize the value of the correlated hopping we introduce dimensionless parameters $\tau_{1}=T_{1}(i j) /\left|t_{i j}\right|, \tau_{2}=T_{2}(i j) /\left|t_{i j}\right|$. 


\section{Ferromagnetism in the case of weak intra-atomic interaction}

The single-particle Green function satisfies the equation

$$
\begin{aligned}
(E- & \mu)\left\langle\left\langle a_{p \sigma} \mid a_{p^{\prime} \sigma}^{+}\right\rangle\right\rangle_{E}=\frac{\delta_{p p^{\prime}}}{2 \pi}+\sum_{i} t_{i p}(n)\left\langle\left\langle a_{i \sigma} \mid a_{p^{\prime} \sigma}^{+}\right\rangle\right\rangle_{E} \\
& +\sum_{i} T_{2}(i p)\left\langle\left\langle a_{p \bar{\sigma}}^{+} a_{p \bar{\sigma}} a_{i \sigma} \mid a_{p^{\prime} \sigma}^{+}\right\rangle\right\rangle_{E}+\sum_{i} T_{2}(i p)\left\langle\left\langle a_{i \bar{\sigma}}^{+} a_{i \bar{\sigma}} a_{i \sigma} \mid a_{p^{\prime} \sigma}^{+}\right\rangle\right\rangle_{E} \\
& +2 \sum_{i} T_{2}(i p)\left\langle\left\langle a_{p \bar{\sigma}}^{+} a_{i \bar{\sigma}} a_{i \sigma} \mid a_{p^{\prime} \sigma}^{+}\right\rangle\right\rangle_{E}+U\left\langle\left\langle n_{p \bar{\sigma}} a_{p \sigma} \mid a_{p^{\prime} \sigma}^{+}\right\rangle\right\rangle_{E} \\
& +\frac{1}{2} J \sum_{j \sigma^{\prime}}\left(\left\langle\left\langle a_{j \sigma^{\prime}}^{+} a_{p \sigma^{\prime}} a_{j \sigma} \mid a_{p^{\prime} \sigma}^{+}\right\rangle\right\rangle_{E}-\left\langle\left\langle a_{j \sigma^{\prime}}^{+} a_{j \sigma} a_{p \sigma^{\prime}} \mid a_{p^{\prime} \sigma}^{+}\right\rangle\right\rangle_{E}\right)
\end{aligned}
$$

Let us consider the system at weak intra-atomic Coulomb interaction $(U<$ $\left.\left|t_{i j}(n)\right|\right)$. Then we can take into account the electron-electron interactions in the Hartree-Fock approximation:

$$
\begin{aligned}
& \left\langle\left\langle a_{i \bar{\sigma}}^{+} a_{i \bar{\sigma}} a_{j \sigma} \mid a_{p^{\prime} \sigma}^{+}\right\rangle\right\rangle_{E} \simeq\left\langle a_{i \bar{\sigma}}^{+} a_{i \bar{\sigma}}\right\rangle\left\langle\left\langle a_{j \sigma} \mid a_{p^{\prime} \sigma}^{+}\right\rangle\right\rangle_{E}, \\
& \left\langle\left\langle a_{i \bar{\sigma}}^{+} a_{j \bar{\sigma}} a_{i \sigma} \mid a_{p^{\prime} \sigma}^{+}\right\rangle\right\rangle_{E} \simeq\left\langle a_{i \bar{\sigma}}^{+} a_{j \bar{\sigma}}\right\rangle\left\langle\left\langle a_{i \sigma} \mid a_{p^{\prime} \sigma}^{+}\right\rangle\right\rangle_{E} .
\end{aligned}
$$

We assume that averages $\left\langle a_{i \sigma}^{+} a_{i \sigma}\right\rangle=n_{\sigma}$ are independent of the number of the site, i.e. we limit ourselves to a uniform charge and electronic magnetic moment distribution (the problems of antiferromagnetic and charge orderings will be studied elsewhere).

The approximation (3.2) corresponds to the condition of weak electron-electron interaction, where doubly occupied states can be taken into account by the effective mean field depending on the electron concentration and correlated hopping integral.

After the transition to a Fourier representation we obtain for the Green function

$$
\left\langle\left\langle a_{p \sigma} \mid a_{p^{\prime} \sigma}^{+}\right\rangle\right\rangle_{\mathbf{k}}=\frac{1}{2 \pi} \frac{1}{E-E_{\sigma}(\mathbf{k})},
$$

where the energy spectrum is

$$
E_{\sigma}(\mathbf{k})=-\mu+\beta_{\sigma}+n_{\bar{\sigma}} U-z n_{\sigma} J+t(n \sigma) \gamma(\mathbf{k}),
$$

here the spin-dependent shift of the band center is

$$
\beta_{\sigma}=\frac{2}{N} \sum_{i j} T_{2}(i j)\left\langle a_{i \bar{\sigma}}^{+} a_{j \bar{\sigma}}\right\rangle,
$$

$\gamma(\mathbf{k})=\sum_{\mathbf{R}} \mathrm{e}^{\mathrm{i} \mathbf{k} \mathbf{R}}$ and the spin and concentration dependent hopping integral is

$$
t(n \sigma)=t(n)+2 n_{\bar{\sigma}} T_{2} .
$$

The concentration of electrons with spin $\sigma$ is

$$
n_{\sigma}=\int_{-\infty}^{+\infty} \rho(\epsilon) f(\epsilon) \mathrm{d} \epsilon .
$$


Here $\rho(\epsilon)$ is the density of states, $f(\epsilon)$ is the Fermi distribution function. Let us assume the rectangular density of states:

$$
\rho(\epsilon)=\frac{1}{N} \sum_{\mathbf{k}} \delta(\epsilon-\epsilon(\mathbf{k}))=\frac{1}{2 w} \theta\left(\epsilon^{2}-w^{2}\right) .
$$

Then in the case of zero temperature we obtain:

$$
n_{\sigma}=\int_{-\infty}^{+\infty} \rho(\epsilon) \theta\left(-E_{\sigma}(\epsilon)\right) \mathrm{d} \epsilon=\frac{1}{2 w} \int_{-w}^{w} \theta\left(-E_{\sigma}(\epsilon)\right) \mathrm{d} \epsilon=\frac{1}{2 w} \int_{-w}^{\epsilon_{\sigma}} \mathrm{d} \epsilon
$$

where $\epsilon_{\sigma}=w\left(2 n_{\sigma}-1\right)$. The value $\epsilon_{\sigma}$ is the solution of the equation $E_{\sigma}(\epsilon)=0$ from which we obtain $\epsilon_{\sigma}=\mu_{\sigma} / \alpha_{\sigma}$, where $\mu_{\sigma}=\mu-\beta_{\sigma}+z n_{\sigma} J-n_{\bar{\sigma}} U$ and $\alpha_{\sigma}=1-2 \tau_{2} n_{\bar{\sigma}}$.

The system parameters are related by the equation

$$
m(z J+U)+\beta_{\downarrow}-\beta_{\uparrow}=2 m\left(1-\tau_{2}\right) .
$$

The shift of the band center is obtained from

$$
\beta_{\sigma}=\frac{2}{N} \sum_{i j} T_{2}(i j)\left\langle a_{i \bar{\sigma}}^{+} a_{j \bar{\sigma}}\right\rangle=-\frac{\tau_{2}}{2 w} \int_{-w}^{\epsilon_{\sigma}} \epsilon \mathrm{d} \epsilon=-\tau_{2} w n_{\bar{\sigma}}\left(n_{\bar{\sigma}}-1\right) .
$$

One can see that

$$
\beta_{\downarrow}-\beta_{\uparrow}=2 \tau_{2} m w(1-n) .
$$

From (3.10) and (3.12) we obtain the condition of ferromagnetic ordering realization:

$$
\frac{z J+U}{2 w}+\tau(2-n)>1
$$

Here we use the notation

$$
w=w(n)=z\left|t_{0}\right|\left(1-\tau_{1} n\right)
$$

where $t_{0}$ is the band hopping integral, $z$ is the number of nearest neighbours, $\tau=$ $\tau_{2} /\left(1-n \tau_{1}\right)$.

In the figures 1,2 the critical value of exchange integral at which the ferromagnetic ordering occurs is plotted as a function of correlated hopping. The values above the line correspond to the ferromagnetic state of the system, below the line correspond to the paramagnetic state. One can see that in the less than half-filled band, correlated hopping leads to the stabilization of ferromagnetism as well as inter-atomic exchange interaction and intra-atomic Coulomb interaction; the larger the electron concentration $n$, the smaller the critical value of the exchange integral for the occurrence of ferromagnetism. These results of ours are in accordance with the results of reference [9]. 


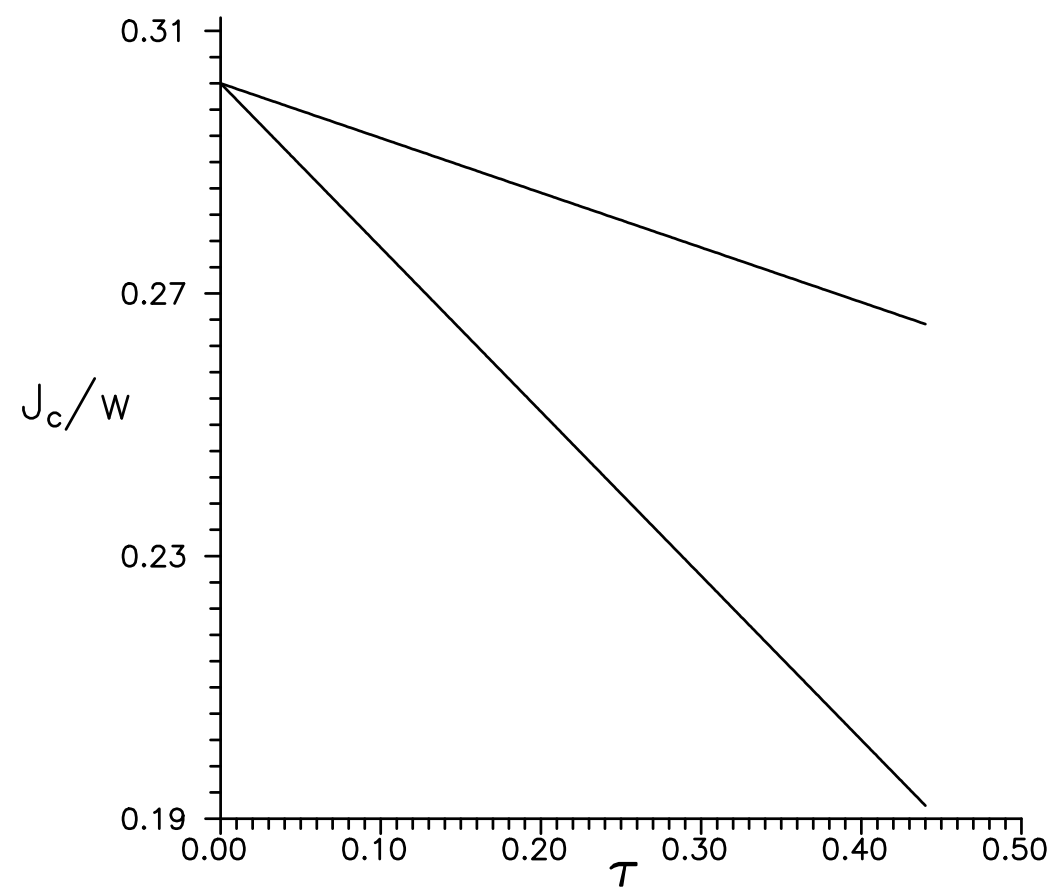

Figure 1. The critical value of the exchange integral as a function of $\tau=\tau_{2}$ at correlation strength $U / w=0.2$ and $\tau_{1}=0$ : lower curve corresponds to $n=1.5$; upper curve corresponds to $n=0.5$.

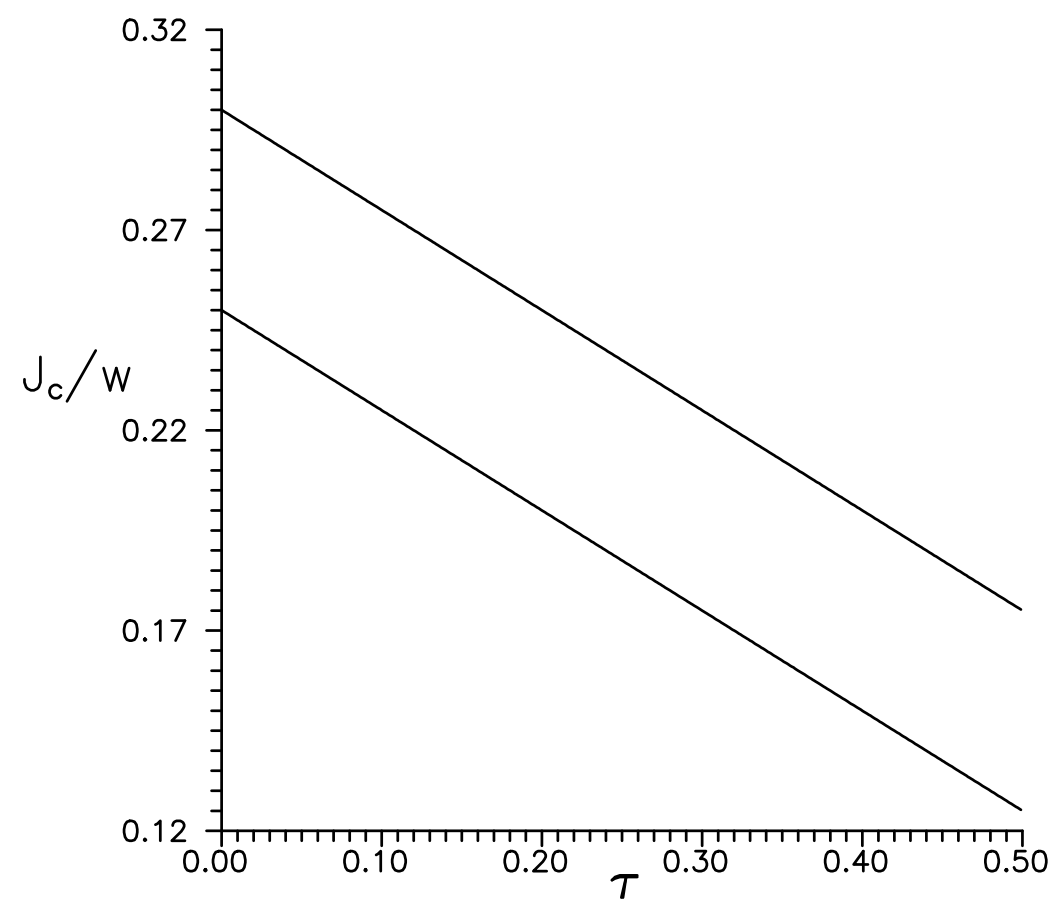

Figure 2. The critical value of the exchange integral as a function of $\tau=\tau_{2}$ at electron concentration $n=0.5$ and $\tau_{1}=0.5$ : lower curve corresponds to $U=0.5$; upper curve corresponds to $U=0.2$. 


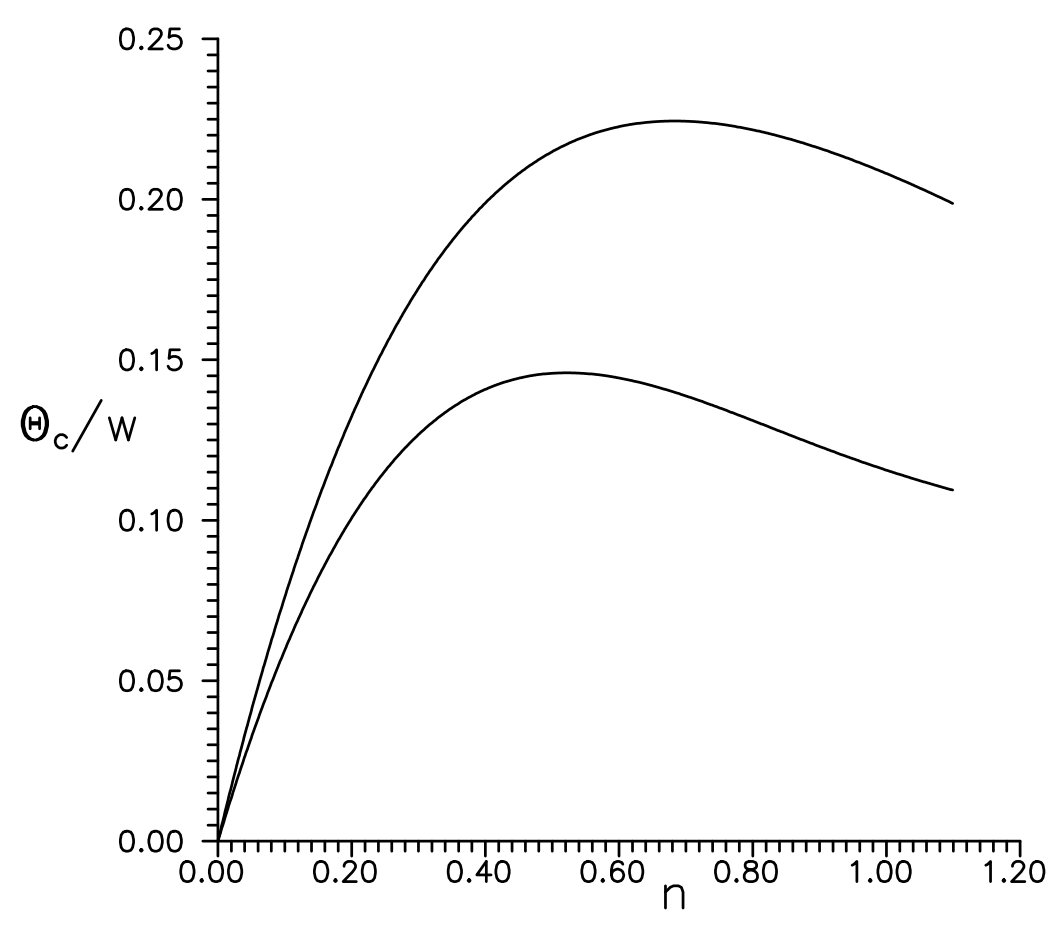

Figure 3. The dependence of Curie temperature on electron concentration $n$; $\tau_{1}=\tau_{2}=0.5$. The lower and upper curves correspond to $U / w_{0}=0.6$ and 0.9 respectively.

In the case of non-zero temperatures the equation for magnetization is

$$
\exp \left(\frac{-m J_{\text {eff }}}{\theta}\right)=\frac{\operatorname{sh}\left[\left(1-\mathrm{n}_{\uparrow}\right) \alpha_{\uparrow} \mathrm{w} / \theta\right] \operatorname{sh}\left[\mathrm{n}_{\downarrow} \alpha_{\downarrow} \mathrm{w} / \theta\right]}{\operatorname{sh}\left[\left(1-\mathrm{n}_{\downarrow}\right) \alpha_{\downarrow} \mathrm{w} / \theta\right] \operatorname{sh}\left[\mathrm{n}_{\uparrow} \alpha_{\uparrow} \mathrm{w} / \theta\right]}
$$

where the effective exchange integral is

$$
J_{\mathrm{eff}}=z J+U+2 z T_{2}(1-n)
$$

The expression for Curie temperature can be obtained from equation (3.15) at $m \rightarrow 0$. If $\tau_{1}=\tau_{2}=0$ then we obtain

$$
\theta_{\mathrm{C}}=\frac{w_{0}}{2} \operatorname{arcth}^{-1}\left(\frac{2 w_{0}}{z J+U}\right)
$$

If $\tau_{1}=\tau_{2}=1 / 2$ then

$$
\theta_{\mathrm{C}}=\frac{w_{0} n(2-n)}{4} \operatorname{arcth}^{-1}\left(\frac{w_{0}}{z J+U+(1-n) w_{0}}\right)
$$

The concentration dependence of Curie temperature given by the last equation is plotted in figure 3. It is important that at some values of parameters the Curie temperature can increase with the decrease of the electron concentration. 


\section{Discussion and conclusions}

The analysis obtained in this paper for the energy spectrum (3.4) shows that both the intra-atomic Coulomb repulsion $U$ and the exchange integral $J$ favour spin polarization. Furthermore, accounting for the correlated hopping leads to a spindependent shift of the band center and to a band narrowing, which also give rise to ferromagnetism. These results are in accordance with the results of reference [9].

The peculiarities of the energy spectrum (3.4) lead to a concentration dependence of the Curie temperature. In particular, the concentration dependent shift (3.12) of the band centers at $n<1$ is positive, at $n>1$ is negative. According to this fact the Curie temperature at decreasing $n$ from 1 can increase and at $n>1$ can decrease. The obtained dependence qualitatively agrees with the experimental data on $\mathrm{Fe}_{x} \mathrm{Co}_{1-x} \mathrm{~S}_{2}$ [10]. The proposed approach can be extended to all values of $n$, and the peculiarities of ferromagnetic ordering in $\mathrm{Co}_{x} \mathrm{Ni}_{1-x} \mathrm{~S}_{2}$ where the concentration changes from 1 to 2 can be explained.

In conclusion, taking into consideration both correlated hopping and inter-atomic exchange interaction essentially modifies the properties of the model and favours ferromagnetic ordering.

\section{References}

1. Rado G.T., Suhl H. Magnetism. New-York, Academic Press, 1966.

2. Friedel J. Physics of Metals. Cambridge, Cambridge University Press, 1969.

3. Gutzwiller M.C. Effect of correlation on the ferromagnetism of transition metals. // Phys. Rev. Lett, 1963, vol. 10, p. 159-162.

4. Kanamori J. Electron correlation and ferromagnetism of transition metals. // Prog. Theor. Phys, 1963, vol. 30, No. 3, p. 275-289.

5. Strack R., Vollhardt D. Exact results on ferromagnetism in correlated electron systems. // J. Low Temp. Phys, 1995, vol. 99, No. 3/4, p. 385-396.

6. Vollhardt D., Blümer N., Held K., Kollar M., Schlipf J., Ulmke M. Non-perturbative approaches to magnetism in strongly correlated electron systems. // Z. Phys. B, 1997, vol. 103, p. 283-292.

7. Tasaki H. From Nagaoka's ferromagnetism to flat-band ferromagnetism and beyond. // Progr. Theor. Phys, 1998, vol. 99, No. 4, p. 489-548.

8. Hirsch J.E. Superconductivity and hydromagnetism. // Physica B, 1990, vol. 163, p. 291-298.

9. Amadon J.C., Hirsch J.E. Metallic ferromagnetism in a single-band model: effect of band filling and Coulomb interactions. // Phys. Rev. B, 1996, vol. 54, No. 9, p. 63646375 .

10. Jarrett H.S., Cloud W.H., Bouchard R.J., Frederick C.G., Gillson J.L. Evidence for itinerant d-electron ferromagnetism. // Phys. Rev. Let, vol. 21, No. 9, p. 617-620.

11. Didukh L. On ferromagnetic ordering in the narrow conduction bands. - In: The Electronic Structure and Physical and Chemical Properties of Refractory Compounds. Kyiv, Naukova Dumka, 1980, p. 32-37.

12. Hubbard J. Electron correlations in narrow energy bands. // Proc. Roy. Soc, 1963, vol. A276, No. 1369, p. 238-257. 
13. Nagaoka Y. Ferromagnetism in a narrow, almost half-filled $s$-band. // Phys. Rev., 1966, vol. 147, No. 1, p. 392-405.

14. Didukh L.D. On the accounting of correlation effects in the narrow conduction bands. // Fiz. Tverd. Tela, 1977, vol. 13, No. 8, p. 1217-1222 (in Russian).

15. Didukh L. A modified form of polar model of crystal. // Cond. Matt. Phys, 1998, vol. 1, No. 1(13), p. 125-144.

16. Didukh L. A model of the narrow-band material with the electron-hole asymmetry. // Journ. of Phys. Stud., 1997, vol. 1, No. 2, p. 241-250 (in Ukrainian).

17. Campbell D. K., Tinka Gammel J., Loh E.Y. Modelling electron-electron interactions in reduced-dimensional materials: bond-charge Coulomb repulsion and dimerization in Peierls-Hubbard models. // Phys. Rev. B, 1990, vol. 42, No. 1, p. 475-492.

\section{Феромагнетизм в узагальненій моделі Габбарда}

\section{Л.Дідух, О.Крамар, Ю.Скоренький}

Тернопільський державний технічний університет імені І. Пулюя, кафедра фізики, 46001 Тернопіль, вул. Руська, 56

Отримано 14 серпня 2000 р.

В роботі розглядається можливість реалізації феромагнітного впорядкування в узагальненій моделі Габбарда з корельованим переносом. Обгрунтована необхідність врахування міжатомної обмінної взаємодії електронів, що є важливим фактором при розгляді феромагнетизму у вузькозонних матеріалах. У випадку слабкої внутрішньоатомної взаємодії знайдено умови реалізації феромагнітного впорядкування, отримано вирази для намагніченості, температури Кюрі, залежні від параметрів системи. Вказано на важливу роль корельованого переносу, який суттєвим чином модифікує умови виникнення феромагнітного впорядкування.

Ключові слова: феромагнітне впорядкування, корельований перенос, обмінна взаємодія, намагніченість, температура Кюрі

PACS: $71.10 . F d, 71.30 .+h, 71.27 .+a$ 